\title{
An Optimal Control Problem for Stereotactic Neurosurgery
}

\author{
Kathrin Flaßkamp* Karl Worthmann*** \\ Christoph Greiner-Petter ${ }^{* *}$ Christof Büskens * \\ Thomas Sattel ${ }^{* *}$ \\ * Center for Industrial Mathematics, University of Bremen \\ (e-mail: kathrin.flasskamp@uni-bremen.de, \\ bueskens@math.uni-bremen.de) \\ ** Department of Mechanical Engineering, Technische Universität \\ Ilmenau \\ (e-mail: $\{$ christoph.greiner-petter/thomas.sattel\}@tu-ilmenau.de) \\ *** Institute for Mathematics, Technische Universität Ilmenau \\ (e-mail: karl.worthmann@tu-ilmenau.de)
}

Keywords: Trajectory and Path Planning, Modeling for Control Optimization, Telerobotics, Robots manipulators, Control in Neuroscience, Mechatronic Systems Modeling

\section{INTRODUCTION}

In typical stereotactic neurosurgery, an entry point on the skullcap is determined from which the target region inside the brain is approached on a straight line, see, e.g. Rahman et al. (2009). MRT or CT data provide information on one or multiple target points in the brain, which the cannula has to reach during operation, as well as on critical regions inside the brain that should not be penetrated. Stereotactic neurosurgery requires careful a priori design and planning of cannula trajectories.

Here, we focus on a novel approach to mitigate negative side effects due to the penetration of brain tissue. To this end, we propose the usage of a cannula that is composed of several pre-curved nickel-titanium tubes with decreasing diameter such that their construction allows for intertwining, see Fig. 1. Then, surgery planning includes choosing an entry point on the skullcap, the cannula design, e.g. how many and which type of cannula, and the computation of control commands for the individual tubes of the cannula. Using actively deformable cannulas offers the possibility to reach multiple target points through one entry point and may even allow to reach a target point in view off critical regions inside the brain.

However, actively deformable cannulas give raise to an infinite number of possible tube trajectories, such that it is inevitable to support surgery planning by mathematical optimization.

\section{MODELING \& OPTIMIZATION}

We model the design and planning problem as a constrained optimization problem. The optimization variables can be partitioned into design and control variables, see Fig. 1. While the former have to be fixed before the cannula is built, the latter can be adjusted later on, e.g. after parameter identification or by model-based control. Finding steering curves for the individual tubes of the cannula can be modeled as an optimal control problem; details are presented in the following.

Kinetic/Kinematic Model: We develop kinetic and kinematic models, in which the configuration of the cannula and its history is decisive while the actual speed of realizing that path can be ignored. Hence, time does not play a role. However, in order to take the configuration and its history properly into account, we artificially introduce a time parameter. Within robotics terminology, our planning problem is thus between a path and a trajectory planning problem. Ordinary differential equations are derived, whose solution trajectories are absolutely continuous, which ensures smoothness of the computed path for the cannula.

Additional Constraints: Both technical boundary conditions, such as parameter ranges, mechanical material characteristics (cf. Greiner-Petter and Sattel (2017)), and medical aspects, such as intolerable lateral movements of the cannula or the structure of the brain, have to be taken into account as equality or inequality constraints on tube trajectories.

Objective Function: We identified several different objective functions of interest. The length of the fully extended cannula, i.e. when the target region is reached, should be minimized, which corresponds to a classical Mayer-term in an optimal control problem. Moreover, torsion of the elastic tubes may cause violations of the follow-the-leader behavior, which leads to undesired lateral movements of the cannula and, thus, severe damage of tissue and therefore, has to be minimized. A similar behavior can be observed due to hysteresis.

These objectives are potentially conflicting optimization criteria. Thus, they have to be considered in a multiobjective optimization framework and e.g. handled by scalarization methods. A set of Pareto optimal solutions, i.e. optimal compromises of the considered optimization criteria, can greatly support decision making by allowing 


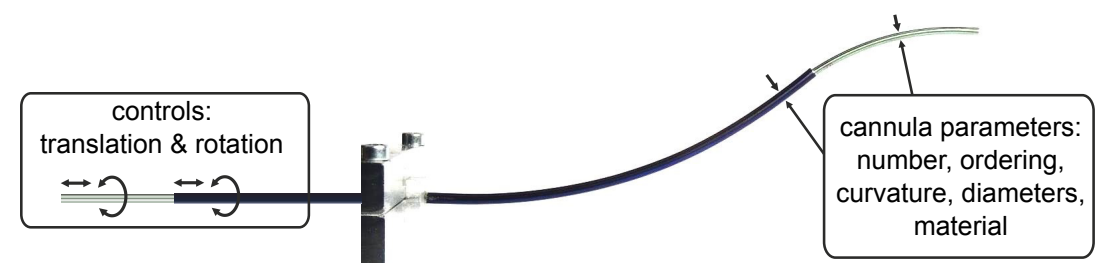

Fig. 1. Picture of surgical instrument with optimization variables indicated: parameters of the cannula and controls.

the surgeon to choose in dependence of the individual patient.

\section{RELATED WORK}

State-of-the-art surgery techniques are based on straight cannulas that are steered via a stereotactic frame. However, recently, research has been done on actively deformable cannulas which provide more degrees of freedom in design and control Burgner-Kahrs et al. (2015). In this case, the mechanical model gains complexity, since interaction between the tubes have to be considered GreinerPetter and Sattel (2017).

Trajectory planning for medical applications is often addressed by stochastic, sampling-based, or heuristic optimization approaches, e.g. the Nelder-Mead method in Bergeles et al. (2015) or rapidly exploring roadmaps in Alterovitz et al. (2011); Torres and Alterovitz (2011). Further, Monte-Carlo Simulations have been used in BurgnerKahrs et al. (2014) for workspace characterization. Approaches to collision avoidance for brain regions have been considered in Lyons et al. (2009, 2010); Alterovitz et al. (2011); Torres and Alterovitz (2011). In Anor et al. (2011), simultaneous trajectory planning and cannula design is presented. However, the approach does not use gradient information but pattern search methods. Global solutions are obtained via varied initial guesses. Optimal planning for actively deformable cannulas by gradient-based techniques has only been considered for simple kinematic cannula models in Lyons et al. (2009).

\section{IMPLEMENTATION \& OUTLOOK}

Our approach to the design and planning problem of actively deformable cannula is to use model-based optimization, more concretely, gradient-based nonlinear optimization techniques. The optimal control problem is discretized by transcription methods, see, e.g. Gerdts (2011). Medical and technical constraints are added to the problem.

The development of structure-exploiting efficient optimization software, e.g. WORHP, Büskens and Wassel (2012), nowadays allows to use gradient-based optimization techniques, as opposed to sampling heuristics, even in highly complex applications with many variables and constraints. Here, local optimality of solutions can be guaranteed and the numerical solution offers further information in terms of sensitivities, which can be used to study robustness properties of solutions, for instance. Robustness is of utmost importance for the considered application in order to cope with modeling errors and external disturbances.

In future, this contribution will be part of a trajectory planning tool for a mechatronic instrument for stereotactic neurosurgery.

\section{REFERENCES}

Alterovitz, R., Patil, S., and Derbakova, A. (2011). Rapidly-exploring roadmaps: Weighing exploration vs. refinement in optimal motion planning. In IEEE Int. Conf. on Rob. and Autom., 3706-3712.

Anor, T., Madsen, J.R., and Dupont, P. (2011). Algorithms for design of continuum robots using the concentric tubes approach: A neurosurgical example. In IEEE Int. Conf. on Rob. and Autom., 667-673.

Bergeles, C., Gosline, A.H., Vasilyev, N.V., Codd, P.J., del Nido, P.J., and Dupont, P.E. (2015). Concentric tube robot design and optimization based on task and anatomical constraints. IEEE Trans. Rob., 31(1), 67-84.

Burgner-Kahrs, J., Gilbert, H., Granna, J., Swaney, P., and Webster, R. (2014). Workspace characterization for concentric tube continuum robots. In IEEE/RSJ Int. Conf. on Intell. Robots and Syst., 1269-1275.

Burgner-Kahrs, J., Rucker, D., and Choset, H. (2015). Continuum robots for medical applications: A survey. IEEE Trans. Rob., 31(6), 1261-1280.

Büskens, C. and Wassel, D. (2012). The ESA NLP solver WORHP. In Modeling and optim. in space eng., 85-110. Springer.

Gerdts, M. (2011). Optimal Control of ODEs and DAEs. De Gruyter.

Greiner-Petter, C. and Sattel, T. (2017). On the influence of pseudoelastic material behavior in planar shapememory tubular continuum structures. Smart Materials and Structures.

Lyons, L., Webster, R., and Alterovitz, R. (2009). Motion planning for active cannulas. In IEEE/RSJ Int. Conf. on Intell. Robots and Syst., 801-806.

Lyons, L., Webster, R., and Alterovitz, R. (2010). Planning active cannula configurations through tubular anatomy. In IEEE Int. Conf. on Rob. and Autom., 20822087.

Rahman, M., Murad, G., and Mocco, J. (2009). Early history of the stereotactic apparatus in neurosurgery. Neurosurg. Focus, 27(3).

Torres, L. and Alterovitz, R. (2011). Motion planning for concentric tube robots using mechanics-based models. In IEEE/RSJ Int. Conf. on Intell. Robots and Syst., 5153-5159. 